\title{
Experiment Test Design of Consumption Accompanying Behavior in VR Environment
}

\author{
Chunying Cui \\ School of Economics \&Management \\ Wenhua College \\ Wuhan, China \\ dannycui@163.com
}

\author{
Yunqian He \\ School of Economics \&Management \\ Wenhua College \\ Wuhan, China
}

\begin{abstract}
This paper mainly used the virtual 3D glasses technology to make an experimental test about the consumer behavior, analyzed how to build up a VR test-based platform, and explored an effective way of making the behavioral experiments. The experiment's design is based on constructing a PC VR environment and choosing the designated 3D virtual consumption accompanying scenes. Under the observations of the controlling group, the paper gets the effective experimental feedback and analyzes the linkage and relationships between the customers' psychological activity and their behavior motivation.
\end{abstract}

Keywords-virtual reality; consumption accompanying; experiment method; scene simulation

\section{INTRODUCTION}

With the combination of virtual reality hardware and experimental environment, the development of the virtual reality (VR) technology has made the teaching and research experiments possible. From 2015 on, the booming of virtual glasses hardware, such as Oculus, Playstation VR, HTC Vive and etc. has provided a hardware foundation for the design of the teaching experimental environment. However, the basement of the hardware commercial production platform couldn't support the software environment field, and thus, the platform can't go supporting the on-entertained applications like customer behavior analyzing, virtually designing, educating, and etc. The economics experimental education based on VR devices are rare; meanwhile, the customer behavior experimental activities need the innovative thoughts provided by VR technology.

\section{THE DESIGN PRINCIPLES OF THE EXPERIMENTAL PLATFORM IN THE VIRTUAL VISUAL ENVIRONMENT}

\section{A. The Problems of the Traditional Experimental Methods}

Traditional consumer behavior science experimental research methods have obvious defects. There are two types of virtual environment. One is to build the real consumption scenarios for the conduction of simulation and experimental test scenarios behavior simulation which the characteristics and activities observed recording form can be used to analyze the data. The other is the image description to complete real scene structures which makes the person to be tested by consistent imagination to complete the activity.
The problems of building the first class scenario of simulation are the lack of the details of environmental simulation and lack of the capital investment. In addition, the environmental construction of the high cost of the transformation experimental difficulties makes tests less repeated. The second class of virtual environment, simulation, and test environment embedded immersed feeling which cause the failure. Moreover, the possibility of producing the game standard is very small, and the individual cognitive differences cause the laboratory environment seriously inconsistent.

The existing design methods - consumer behavior study is mainly based on Maslow's hierarchy of needs theory, in consumption with areas of design from the 1970s hand-held scanner experimental methods. The scene we used is based on the supermarket open shelf shopping scene, with equipped hand-held commodity scanner, the shopping behavior of consumers could be tracked and recorded. Subjects with commodity pricing scanning and electromagnetic logging information can easily locate the positions of the consumer hot spot area and the purchase of hot commodities.

As a new method of experimental research, experimental study of the consumer behavior from the tools has evolved. Observation through biology and medicine are applied in the experimental test, using the pupil observation, CT scan of brain, eye movement instrument testing instrument and equipment implementation of the innovative experiment design.

\section{B. Consumption Accompanying and Group Psychology}

After individual consumer behavior scans were analyzed, scholars started to pay attention to conformity consumption psychology and the effect of study. Herd mentalities in subjects with consumer psychological accompany research in behavioral psychology gives analysis of the interpretation of some of the classic. Kassarijian [1] pointed out that, consumer personality is not with spatial and temporal scenarios change and static, it should be a dynamic concept - individual consumption to changes with time and space environment and the customary way. This opens the door to a stimulus response (S-R) research paradigm and learning theory research. Many experiments showed that a consumer behavior is the environmental stimulus to make a kind of behavior inertia of the adaptive response. And the environment of learning and adaptation can gradually form the consistency of individual consumer behavior. On this basis, from individual to group 
related consumer behavior research to the related groups and opinion leaders influence, we are going to explain groups herd behavior and behavior model.

In the transition from the individual consumer behavior to the consumer behavior of a unique consumption phenomenon, individual consumers in the consumption and purchasing activities with similar acts of other familiar or unfamiliar common individual consumption have strengthened the consumption motives or formed a dependence on the behavior of others state in the process of consumption. It continues to be daily observed, in accordance with the individual to group cognitive and behavioral practice rules. Correspondingly, opinion leader mode is a claim of the authority influence audience consistency judgment, resulting in consistent behavior of the consumer behavior.

After analyzing the past consumer behavior study between conformity and consumer individual action research, it could be found that there is a lack of consumer study. In the field of behavioral experiments, consumption is easy to do daily observation and interpretation, but difficult to pass the scene to unfold the imaginary research.

\section{THE BUILDING OF EXPERIMENTAL DESIGN}

\section{A. The Experiment Design Logic with the Consumption of VR}

Consumer decision-making process is just like the distinction between women's procurement purchase activity and casual shopping activity, which are two kinds of disparate behaviors. In consumption with the process, unaccompanied personnel psychological resonance constitutes the effect to accompany the differences. This resonance can be explicit information communication, such as talking; it can also be implicit information communication, such as state or action eye contact.

In the consumer with the experimental observations of the behavior, hidden information communication often constitute consumption to accompany state herd mentality to produce consistency expected normal reaction, known as Shun to conformance testing. And the explicit information communication, especially language communication, often changes along with the consistency of the impact factors.

The experimental verification is the consumer with the implicit communication information, such as common consumer experience exists (or a relatively static activities) to bring the affirmative judgment and for consumers to the original consumer behavior of Shun to consistent effect. These experiences include pain and suffering, not a subjective happy acts, and in consumer behavior, people are in obvious incentive pleasure. Evidence from brain CT scan results, from shopping consumer behavior of physiological science stimulation prompts brain dopamine secretion, bringing the effect similar to the state of brain activity. The physiological scanning analysis supports that people in the shopping decision are irrational assumptions. Irrational consumption is enhanced or weaken? Whether the groups of non-rational anterograde spread? This has become the consumer with experiments to explore the future direction.
The main purpose of the experimental design attempts to solve the basic problem with the state of the companion which is for consumers to be a psychological hint and dependence. Because in herd behavior, familiar with peer behavior consistency and trust in the tacit understanding will effectively enhance the consistency of the herd, thus the tendency of stranger behavior will be the same.

\section{B. The Application of VR Technology in the Design Platform}

Marketing research on population consistency expected experimental study showed that the herd mentality is reflecting a combination of cognitive multimedia content from scattered to focus, from single modal perception to the multimodal. This study proposed a consumer with a simple design, create a kind of instinct effect of experience consumption environment by VR technology and experimental framework, using the 3D visually and acoustically technology, providing the simulation of consumer experience operating environment. Through the experimental method to test subjects to inform the state and informed by state, and then possibly, consumer with a psychological dependence and consistency of action is expected.

The hardware and software of the virtual experiment framework are as follows.

- Hardware environment: PC with i3-4170CPU,8G DDR RAM,120G SSD, AMD R9-290 4GB graphic acceleration card, 3Glasses VR output.

- Software environment: Windows 8.1 OS X64 Enterprise Edition, DirectX 11, 3Glasses SDK 2.0.

The first problem is the choice of the VR helmet. In the market of VR helmet systems, only 3Glasses products DK2 can achieve $1920 * 1080$ or higher resolution. The resolution of DK2 is as high as $2560 * 1440$. Through comparing Samsung Gear VR, Google Oculus and 3Glasses under the same VR picture display effect, based on 1080p products obvious grainy, we find that VR scene immersion sense DK2 developers insufficient.

Secondly, according to the requirements of the hardware and software of the VR helmet, operating system must be to achieve $2 \mathrm{~K}$ resolution $3 \mathrm{D}$ acceleration and $4 \mathrm{~K}$ resolution panoramic video playback smooth. This requirement makes graphics operation ability of the whole system strong enough. We choose the AMD R9-290 with 4G RAM.

Building a simulation environment, in view of the present stage VR scene and immersed sense and strong sense of participation, and interactive and touch of weak, we construct a brain secretion of epinephrine stimulation of the scene to replace the brain dopamine stimulated secretion of scene. We design the consumer scene for amusement park roller coaster first person perspective $3 \mathrm{D}$ virtual scene and determine the angle of view for the first operation roller coaster positive open view, 30 seconds speed of the impact of video rendering process, including main ramp through the gravity acceleration test, vertical rolling test, kinetic energy and potential energy conversion of the second gradient repeated testing, and rotational tumbling test. 
Through using the SDK Development of VR scene package only the visual interactive scene and sound effects while ignoring the scene in contact of the consumer, we ensure the immersive VR environment. In the VR scene, the acceleration of the roller coaster set for 2.3 acceleration due to gravity effect because in the absence of imitation of the acceleration of gravity force feedback seat, human perception of acceleration is far less than the true acceleration sense so we raise the visual acceleration to reduce the visual impact of the threshold for the secretion of epinephrine.

TABLE I. VR VIDEO TIMELINE

\begin{tabular}{|c|c|}
\hline Timeline & Event \\
\hline 0-8seconds, & Start climbing \\
\hline 9-12 seconds & $\begin{array}{l}\text { Accelerated into the top circled, the first test of } \\
\text { accompany }\end{array}$ \\
\hline $12-17$ seconds & $\begin{array}{l}\text { Main ramp dive, the first gravity test, the stress } \\
\text { response }\end{array}$ \\
\hline $18-20$ seconds & $\begin{array}{l}\text { Pulled up and circled process, the second test of } \\
\text { accompany }\end{array}$ \\
\hline 21-24 seconds & $\begin{array}{l}\text { Vertical rollover test, the first centrifugal } \\
\text { acceleration test }\end{array}$ \\
\hline 25-27 seconds & 90-degree bend, the third test of accompany \\
\hline $28-30$ seconds & Rotating roll, the final test of accompany \\
\hline
\end{tabular}

Test to simulate the common amusement park roller coaster ride process. Roller coaster is very easy to stimulate consumer recreation adrenaline secretion of recreation projects. As a service consumption process, the roller coaster ride is with conspicuous consumption. Project design for ordinary roller coaster overlooking the thrust acceleration of gravity between 1.3 to 2.9 and reaching 1.9 acceleration of gravity have to play a good stimulation effect of epinephrine secretion ride. Then most of the roller coaster consumers go through with the consumer to experience the adrenaline caused panic, and the sudden outbreak of adrenergic stimulation of release pleasure.

This panic and body overload feeling creates the special consumption process; test takers must be a period of tension in the heart of a sudden tension sharing dependency, which is roller coaster project consumption with the first motivation. During peak epinephrine after the intermission, testing and feedback behavior consistency of observation to obtain the approbation of the herd mentality, after acquired dependent. In fact, most of the roller coaster projects retained the passenger seat side by side. The reason is that the real roller coaster experience is full of charm, which has become the foundation of the consumption.

In this process of frequent consumption, personal consumption and group behavior form a close relationship with the consumption situation, which constitute the basic VR scenario we test. We simulate a large acceleration of the virtual roller coaster project and keep the ride around with virtual characters to consumer. Roller coaster on behalf of adrenergic stimulation and human normal consumption stimulation can be observed significantly. Similarly, embodied in the process of consumption and herd consistency, it also will be significantly higher than the ordinary consumption situation. This is the direct cause of the roller coaster project selection of the experimental design.
From the interactive interpretation, the visual stimuli and feedback, the VR scene we constructed can ignore the lack of auditory and tactile interaction. $80 \%$ of the people perceive the external information from visual. If the visual stimuli gets stronger, we must create an interactive, transformed VR immersion from physical aspect to head angle in the simulation process. In this way, the experimental equipment and implementation technology will greatly reduce the difficulty of building VR experiment scenes.

If we analyze from the view of the consumer with real experience, secretion of epinephrine with type of consumer is rare instances of our general cognitive process of consumer behavior. After all, consumers not often use roller coaster project. However, the occasional small probability event often gives us the design inspiration. Because the scene's low level of familiarity, visual impact and true perception, people are awakened by recalling to the adrenaline rapid secretion of panic process, resulting in VR scene of past experience identity. In a real and repeated consumption situation, it is difficult for us to produce the temporal and spatial environment of the mutation and the occasional impact of the consumer instinct.

\section{Experimental Test Process Design}

In order to observe the participants at the time of roller coaster experiments, we have designed a sitting in subjects on the right side of the virtual characters, as a fake, accompanied with the state's partner. The virtual characters can be used as an operating program, selected in the experiment method or disappear.

There are several switch settings, in the virtual image of a companion, also including peer status informed in advance or not. We usually set two subjects who participate in the experiment respectively into the test equipment in different rooms inside, and inform or not inform the subject that virtual character in the virtual world around him is a familiar companion. In addition, we also develop a virtual sound. In the climax of adrenaline dive and roll, we'll open the passenger behind the group screaming, screaming without control which is observed by measuring whether the chain leading to cries are emotional release.

Feedback from the past experience of the riders of roller coaster has shown that each time when they were experiencing the impact of the acceleration of gravity, unprepared subjects were unable to get rid of the picture within the sense of acceleration and velocity field of view of the impact with no time to be associated with the state of observation.

And after experiencing the shock of panic, attention to restore normal subjects tends to observe what others have. According to the similar validation habits later on, we can well design how to observe the sample point.

Experimental observations and method of operation is as follows, $\mathrm{O}$ for observation, $\mathrm{X}$ indicates the operation.
Reference group:
$\begin{array}{lll}\mathrm{O}_{1} & \mathrm{X}_{0} & \mathrm{O}_{2}\end{array}$
Experimental group:
$\begin{array}{lll}\mathrm{O}_{1} & \mathrm{X}_{1} & \mathrm{O}_{2}\end{array}$ 
$\left(\mathrm{O}_{1}\right.$ represents the initial observation, $\mathrm{O}_{2}$ indicates that a return visit after the test.)

$\mathrm{X}_{0}$ operation shows tester using the original video, without a concomitant appearance, $\mathrm{X}_{1}$ represents with. Later we can continue to set $X_{2}$ operation, such as to inform the accompanied virtual character as his companion, control did not inform the companion, with test acquaintances and strangers with the difference; $\mathrm{X}_{3}$, arrange with screaming voice control did not schedule a voice screaming until $X_{n}$.

In reference to the group control experiment, as measured by the pairwise grouping into the experimental environment and the separation of the test, which makes the measurement itself becomes more interesting. Because of the sense of substitution of a virtual character, with the test personnel to inform the measured who sat in the virtual human in his or her right is or is not their own acquaintances, will lead to positive associations of the person under test. This show without state experiments can be through a virtual image, according to network multiplayer role-playing games MMRPG.

Observation plan uses observation record and rhythm of the heart through heart rate records to determine the adrenalin rush, through the observation and the helmet phase anchor point of mobile location records to observe the visual observation attention direction of the subjects. Subject to the VR helmet and 110-degree viewing angle limits fixed IPD problem, the subject's eye could not rotate to change the viewing angle in VR scene, so observing the movement of the head phase is more scientific attention observation modes.

Because the subjects have been informed previously, and in the VR environment can indeed be observed on the right side of the existing consumer with partners of virtual image, the researchers looked at records only measured by observation and turned on the right side of the frequency and occurrence time. They can use frequency data to compare the consumer with reliance situation. Correspondingly, observation can be recorded because of panic and cry, in order to determine the herd mentality caused fear release whether there are significant differences.

\section{EXPERIMENTAL RESULTS AND VERIFICATION}

In a week, we arrange the subjects of the control group before and after the simulation experiment. In consumption based on VR environments with test platform, we have the following test results.

1)The participants' stress response timeline hypothesis is verified that started the scenarios such as diving, subjects too busy to go to the side of visual image. That means VR scene simulation status is close to real, can let the participants have enough sense of immersion. But the trouble is still, as the familiar degree deepening, participates in the repeat test subjects showed amazing sense of curiosity about the VR scene, in the process of roller coaster running, they just ignored the picture, and chose to observe the environment all round.

2)After experiencing for the first time adrenal secretion impact, subjects conformity consistency preference is obvious. Most of the participants chose next to observe with virtual image. The herd mentality is also mentioned in the enquiry of the posttest, a lot of people are trying to view for companion to measure the impact of their degree, which is the psychology direct validation of anchoring effect.

3) Observers who can't observe screaming will be triggered by environmental scream. That is to say, no matter play or not to play with the screams, participants almost don't scream. After interviews, participants said the sound is hard to give them attention.

\section{CONCLUSION}

This paper proposes a VR operation by building a virtual platform, scenario development, and test results indicate that consumption accompanying virtual reality experiment innovative good solution to the shortcomings of traditional experimental, while reducing the cost of experimental testing. In the study of virtual experiment to test consumer attendant, VR platform preliminary teaching and research, the result of controlled experiments and test evaluation of the virtual platform are also satisfactory.

\section{REFERENCES}

[1] Kassarijian, Haroldm H., "Personality and Consumer Behavior: A Review,” Journal of Marketing Research, 1971, 8(Novermber):pp. 409418.

[2] Kassarijian, Harold, and May Jane Sheffet, "Personality and Consumer Behavior: An Update,” in Perspective in Consumer Behavior. $4^{\text {th }}$ ed., Kassarijian, Harold, H. and Thomas S. Robertson, eds, Englewood Cliffs, NJ:Printice-Hall International, Inc. 1991:pp.81-301.

[3] Yau Yuen YEUNG, “A learner-centered approach for training science teachers through virtual reality and 3D visualization technologies:Practical experience for sharing," Conference Paper for The Fourth International Forum on Education Reform, 2004, September.

[4] Zhao Wei, Duan Hong, "Research of Virtual Reality Software," Computer Technology and Development, 2012, 2, Vol (2): pp. 229-233. (In Chinese)

[5] Zhu Xiaowei, "Research on the Design System of Garment Display Based on Process," Research and Exploration in Laboratory, 2016, 4, Vol(35):pp. 150-154. (In Chinese) 